\title{
A diagnostic rule for tuberculous meningitis
}

\author{
Rashmi Kumar, S N Singh, Neera Kohli
}

\begin{abstract}
Diagnostic confusion often exists between tuberculous meningitis and other meningoencephalitides. Newer diagnostic tests are unlikely to be available in many countries for some time. This study examines which clinical features and simple laboratory tests can differentiate tuberculous meningitis from other infections. Two hundred and thirty two children (110 tuberculous meningitis, 94 nontuberculous meningitis, 28 indeterminate) with suspected meningitis and cerebrospinal fluid (CSF) pleocytosis were enrolled. Tuberculous meningitis was defined as positive CSF mycobacterial culture or acid fast bacilli stain, or basal enhancement or tuberculoma on computed tomography (CT) scan with clinical response to antituberculous treatment. Nontuberculous meningitis was defined as positive CSF bacterial culture or Gram stain, or clinical response without antituberculous treatment. Thirty clinical/ laboratory features of patients with tuberculous meningitis and nontuberculous meningitis were compared by univariate and multiple logistic regression analysis. Five features were independently predictive of the diagnosis of tuberculous meningitis $(p<0.007)$ : prodromal stage $\geqslant 7$ days, optic atrophy on fundal examination, focal deficit, abnormal movements, and CSF leucocytes $<50 \%$ polymorphs. When validated on another set of 128 patients, if at least one feature was present, sensitivity was $98.4 \%$ and, if three or more were present, specificity was $98.3 \%$. This simple rule would be useful to physicians working in regions where tuberculosis is prevalent.

(Arch Dis Child 1999;81:221-224)
\end{abstract}

Keywords: tuberculous meningitis; pyogenic meningitis; computed tomography scan

Department of Pediatrics, King George's Medical College, Lucknow, India 226003

R Kumar

S N Singh

Department of Radiodiagnosis, King George's Medical College

N Kohli

Correspondence to: Dr Kumar.

Accepted 15 February 1999
Tuberculous meningitis continues to be an important cause of hospital admissions, death, and neurological disability in children in India. The illness accounts for $1-4 \%$ of total paediatric hospital admissions in different parts of the country. ${ }^{1}$ Tuberculous meningitis is a serious illness which, if not diagnosed and managed early, leads to a high rate of mortality and permanent disabilities.

Today, tuberculous meningitis still poses a diagnostic problem. The reason is that it presents in a similar manner to other meningoencephalitides, partially treated pyogenic meningitis in particular. Definitive diagnosis of tuberculous meningitis can be made by dem- onstration of mycobacteria in cerebrospinal fluid (CSF), by direct staining or culture. However, these tests are time consuming and seldom positive. ${ }^{2}$ Recognising this problem of diagnosis, many newer tests have been developed to diagnose tuberculous meningitis and differentiate it from pyogenic meningitis-for example, enzyme linked immunosorbent assay, bromide partition test, tuberculostearic acid in $\mathrm{CSF}$, adenosine deaminase in CSF, polymerase chain reaction, etc. ${ }^{3}$ However, the sensitivity of these tests is still under study and they are unlikely to be available where they are really needed for at least the next decade. In practice, in India, treatment is started solely on the basis of clinical features and results of simple laboratory tests of CSF and blood. Therefore, we attempted to establish a clinical rule for the diagnosis of tuberculous meningitis, whereby a set of clinical and laboratory findings can be used to "predict" the diagnosis of tuberculous meningitis. Such a rule would be especially useful for clinicians working in remote areas, where laboratory facilities are minimal and diagnosis has to be made on clinical grounds. In addition, identifying a group of children who have certainly (or almost certainly) not got tuberculous meningitis could avoid exposing them to an unnecessary and long course of antituberculous treatment.

\section{Materials and methods}

We conducted our study in the children's wards of King George's Medical College Hospital, Lucknow over a period of 30 months from August 1994 to February 1997. This is a large teaching hospital in the capital city of India's most populous state, Uttar Pradesh, and caters for the poor and seriously ill children from the city and surrounding districts. Children between the ages of 1 month and 12 years admitted with a diagnosis of meningoencephalitis on the basis of history, examination, and pleocytosis in CSF, on three selected days of each week were enrolled and investigated according to a predesigned protocol. A complete physical examination was carried out on admission. Investigations included blood counts, CSF examination for cell count, differential count, protein, sugar, Gram stain, acid fast bacilli (AFB) stain, bacterial and mycobacterial culture, a skin test for tuberculosis, and a chest radiograph. A CT scan was also planned and was usually possible within a week of admission to hospital. The patient's clinical course was carefully recorded. At discharge, the final diagnosis of tuberculous meningitis or nontuberculous meningitis was made on the following criteria.

Tuberculous meningitis was diagnosed if: (1) mycobacterial culture/AFB stain was positive 
Table 1 Clinical and laboratory features in patients with and without tuberculous meningitis (TBM)

\begin{tabular}{|c|c|c|c|c|}
\hline & $\begin{array}{l}T B M \\
(n=110)\end{array}$ & $\begin{array}{l}\text { Non-TBM } \\
(n=94)\end{array}$ & Odds ratio $(95 \% \mathrm{CI})$ & $p$ Value \\
\hline Age (months) & 42.4 & 39.0 & - & $0.56^{\star}$ \\
\hline Prodromal stage (days) & 19.0 & 4.0 & - & $<0.005+$ \\
\hline Weight for age (\%) & 66.2 & 73.4 & - & $<0.005^{\star}$ \\
\hline Haemoglobin $(\mathrm{g} / \mathrm{l})$ & 101 & 100 & - & $0.72^{\star}$ \\
\hline Leucocyte count $\left(10^{9} / 1\right)$ & 9.4 & 10.5 & & $<0.005 t$ \\
\hline DLC ( $\%$ polymorphs $)$ & 66.7 & 71.9 & - & $0.01^{\star}$ \\
\hline CSF cell count & 137.0 & 660.0 & - & $<0.005+$ \\
\hline CSF polymorphs (\%) & 25.0 & 90.0 & - & $<0.005 t$ \\
\hline CSF protein $(\mathrm{g} / \mathrm{l})$ & 1 & 1 & - & $0.38+$ \\
\hline CSF sugar $(\mathrm{g} / \mathrm{l})$ & 0.4 & 0.3 & - & $0.01^{\star}$ \\
\hline Religion Hindu & 86 & 73 & $1.0(0.5$ to 2.1$)$ & 0.936 \\
\hline \multicolumn{5}{|l|}{ Fever grade } \\
\hline Low & 55 & 9 & & \\
\hline Moderate & 38 & 19 & & $<0.005$ \\
\hline High & 17 & 66 & & \\
\hline Vomiting & 73 & 60 & $1.1(0.6$ to 2.1$)$ & 0.82 \\
\hline Convulsions & 80 & 61 & $1.4(0.8$ to 2.7$)$ & 0.29 \\
\hline Focal convulsions & 14 & 16 & $0.6(0.2$ to 1.4$)$ & 0.29 \\
\hline \multicolumn{5}{|l|}{ Convulsion frequency } \\
\hline Nil & 30 & 33 & & \\
\hline $1-3 /$ day & 32 & 22 & - & 0.70 \\
\hline $3-6 /$ day & 27 & 22 & & \\
\hline$>6 /$ day & 21 & 17 & & \\
\hline History of measles & 9 & 4 & $2.0(0.5-8.1)$ & 0.39 \\
\hline History of contact & 46 & 8 & $7.7(3.2-19.3)$ & $<0.005$ \\
\hline Lymphadenopathy & 46 & 14 & $4.1(1.9-8.7)$ & $<0.005$ \\
\hline \multicolumn{5}{|l|}{ Coma grade } \\
\hline Nil & 27 & 25 & & \\
\hline I & 58 & 41 & & 0.58 \\
\hline II & 19 & 22 & & \\
\hline III & 6 & 6 & & \\
\hline Meningeal signs & 95 & 82 & $0.9(0.4$ to 2.3$)$ & 0.98 \\
\hline Fundal optic atrophy & 30 & 2 & 19.7 (4.4 to 123.6$)$ & $<0.005$ \\
\hline Focal deficits & 48 & 10 & $6.5(2.9$ to 15.0$)$ & $<0.005$ \\
\hline Extrapyramidal movements & 35 & 4 & $10.5(3.3$ to 36.9$)$ & $<0.005$ \\
\hline Decerebrate posturing & 26 & 5 & $5.5(1.9$ to 17.4$)$ & $<0.005$ \\
\hline Increased tone & 67 & 54 & $1.1(0.6$ to 2.1$)$ & 0.72 \\
\hline Ankle clonus & 13 & 2 & $6.2(1.3$ to 41.3$)$ & 0.02 \\
\hline Cranial nerve palsies & 25 & 3 & $8.9(2.4$ to 38.6$)$ & $<0.005$ \\
\hline Skin test positive & $36 / 84$ & $4 / 28$ & $4.5(1.3$ to 17.0$)$ & 0.01 \\
\hline Suggestive chest radiograph & $48 / 66$ & $9 / 32$ & $6.8(2.4$ to 19.8$)$ & $<0.005$ \\
\hline
\end{tabular}

For continuous variables (1-10), analysis was either by two sample $t$ test $^{\star}$ and mean values are shown, or by Kruksal-Wallis test $\dagger$ and medians are shown (see methods). For non-continuous variables (11-30) data are numbers of patients with the finding. The $\chi^{2}$ test was used.

CI, confidence interval; DLC, differential leucocyte count; CSF, cerebrospinal fluid.

Table $2 \beta$ Coefficients, $p$ values, and odds ratios (OR) with 95\% confidence intervals (CI) of five factors found to be independently associated with tuberculous meningitis on logistic regression analysi

\begin{tabular}{llll}
\hline & $\beta$ Coeff & $p$ Value & OR (95\% CI) \\
\hline Prodromal stage $\geqslant 7$ days & 2.33 & $<0.005$ & $10.3(3.8$ to 27.9$)$ \\
Fundal optic atrophy & 0.23 & $<0.005$ & $1.3(1.1$ to 1.5$)$ \\
Focal deficit & 1.75 & $<0.005$ & $5.8(1.9$ to 17.7$)$ \\
Extrapyramidal movements & 2.94 & $<0.005$ & $18.9(4.2$ to 84.9$)$ \\
CSF leucocytes $<50 \%$ polymorphs & 3.41 & $<0.005$ & $30.2(9.9$ to 91.6$)$ \\
\hline
\end{tabular}

CSF, cerebrospinal fluid. sample $t$ test, or Kruksal-Wallis test for data that were not normally distributed) using the Epi-info program. Table 1 lists the variables tested. Length of prodromal stage was defined as period between first symptom and first neurological manifestation. Weight was expressed as percentage of expected weight for age. Fever was graded as low $\left(<37.5^{\circ} \mathrm{C}\right)$, moderate $\left(37.5-38.5^{\circ} \mathrm{C}\right.$ ), or high (more than $38.5^{\circ} \mathrm{C}$ ). Frequency of convulsions was divided into 1-3/day, 4-6/day, and > 6/day. A history of measles included the previous six months only. Coma was graded according to Corey et al. ${ }^{5}$ Presence of meningeal signs was defined as neck stiffness, Kernig's or Brudzinsky's sign. Variables with $\mathrm{p}<0.25$ were entered into a logistic regression analysis using the multlr package to obtain $\beta$ coefficients, adjusted odds ratios, and "predictors" of the diagnosis of tuberculous meningitis.

For validation, another set of patients was enrolled in the same way using the same diagnostic (gold standard) criteria. The rule was then applied to them and compared with the gold standard diagnosis in a two by two table to calculate its sensitivity, specificity, and the likelihood ratios for various predictors and their combinations. $\beta$ Coefficients obtained for the five variables in the logistic regression analysis were rounded off and added up to obtain a weighted score for each patient in the validation dataset. Sensitivity and specificity at each score were calculated and these values were used to construct a receiver operator characteristic (ROC) curve and determine the best cut off score. The best cut off point is taken as that closest to the left upper corner of the ROC curve.

\section{Results}

A total of 232 out of the 248 children who satisfied the entry criteria were enrolled. Out of these, 110 had tuberculous meningitis and 94 had non-tuberculous meningitis. A positive CSF culture for Mycobacterium tuberculosis and/or smear for AFB was obtained in a total of 18 patients, whereas the remaining patients in the tuberculous meningitis group were diagnosed on the basis of basal enhancement on CT scan, along with a response to antituberculous treatment.

In 28 patients, the final diagnosis could not be reached on the basis of the diagnostic (gold standard) criteria either because the patient died early or because a CT scan could not be done or was non-confirmatory. These patients were excluded from analysis.

Table 1 shows the clinical and laboratory features in the two groups. Eighteen variables were entered into the logistic regression analysis, of which five were found to have a significant independent association with the diagnosis of tuberculous meningitis. These features are shown in table 2.

The 28 patients who were excluded from analysis included 23 boys and five girls. The mean (SD) age of these 28 patients was 66 (39) months. The median prodromal stage of illness was 13 (range, 2-75) days and 19 had a prodromal stage of seven days or more.

Thirty clinical/laboratory features of tuberculous and non-tuberculous meningitis were compared by univariate analysis $\left(\chi^{2}\right.$ or two 
Table 3 Sensitivity (sens), specificity (spec), and likelihood ratios (LR) at various levels of the predictor variables in the validation dataset

\begin{tabular}{lllllll}
\hline & $\begin{array}{l}\text { Total } \\
(n=128)\end{array}$ & $\begin{array}{l}\text { TBM } \\
(n=66)\end{array}$ & $\begin{array}{l}\text { Non- TBM } \\
(n=62)\end{array}$ & Sens & Spec & LR +ve \\
\hline $\begin{array}{c}\text { One or more predictor } \\
\text { variables present }\end{array}$ & 100 & 65 & 35 & 98.4 & 43.5 & 1.7 \\
$\begin{array}{c}\text { Two or more predictor } \\
\text { variables present }\end{array}$ & 59 & 51 & 8 & 77.2 & 87.0 & 5.9 \\
$\begin{array}{c}\text { Three or more predictor } \\
\text { variables present }\end{array}$ & 37 & 36 & 1 & 54.5 & 98.3 & 30.0 \\
\hline
\end{tabular}

TBM, tuberculous meningitis.

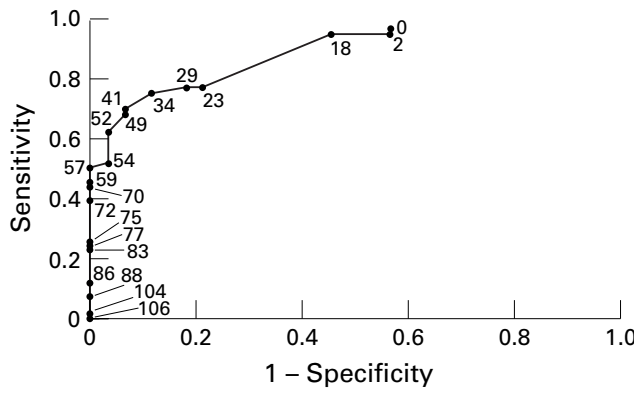

Figure 1 Receiver operator characteristic curve for different scores calculated for each patient in the validation dataset by rounding off and adding the $\beta$ coefficients shown in table 2 (23 for prodromal stage $\geqslant 7$ days, two for fundal optic atrophy, 18 for focal deficits, 29 for extrapyramidal movements, and 34 for cerebrospinal fluid leucocytes $<50 \%$ polymorphs).

Fundoscopy was abnormal in three (papilloedema in two and optic atrophy in one). Focal neurological deficits were found in six patients, and extrapyramidal movements were seen in nine. Median CSF polymorphs was $50 \%$ (range, $0-95 \%$ ), being less than $50 \%$ in 12 patients. Six of these patients had none of the predictors present, 22 had one or more, 13 had two or more, and seven had three or more predictors present. None of these patients had a positive CSF smear or culture for mycobacteria or other bacterial pathogens. Fifteen died in hospital and two left the hospital against medical advice. A CT scan was performed in 16 patients. It was normal in five, and 11 patients had an abnormality that was not supportive of either diagnosis according to our criteria.

The five predictors of the diagnosis of tuberculous meningitis were then validated in the second set of 143 patients, in 128 of whom the diagnosis of tuberculous meningitis (66) or non-tuberculous meningitis (62) could be confirmed using the same gold standards; the remaining 15 were indeterminate. Nine of the 66 TBM patients had a positive CSF mycobacterial culture, but AFB stain and microscopy of CSF was negative in all. Table 3 shows the sensitivity, specificity, and likelihood ratios at various levels of these predictor variables. The $\beta$ coefficients shown in table 2 were rounded off as 23 for prodromal stage $\geqslant 7$ days, two for fundal optic atrophy, 18 for focal deficits, 29 for extrapyramidal movements, and 34 for CSF leucocytes $<50 \%$ polymorphs. Figure 1 shows a ROC curve from the sensitivity and specificity calculated for each score from the validation dataset. The best cut off score was 34 .

\section{Discussion}

Tuberculous meningitis continues to be an important cause of childhood hospital admissions in India. However, at initial presentation it can be confused with other meningoencephalitides, especially partially treated pyogenic meningitis. ${ }^{6}$ Oral antibiotic treatment may prolong the course of the latter, render culture sterile, and even result in lymphocytic predominance in the CSF. On the other hand, in our experience, tuberculous meningitis often presents with a polymorphonuclear reaction in the CSF and blood. In our hospital, in about $30 \%$ of all cases of meningitis, there is diagnostic confusion between tuberculous meningitis and pyogenic meningitis. Yet both are serious life threatening infections requiring early, aggressive treatment to prevent death and disability. Other important differential diagnoses are viral meningitis, encephalitis, and fungal meningitis.

The gold standard for diagnosis used in our study included both investigations and response to treatment and was effective in separating tuberculous meningitis from nontuberculous meningitis. Response to treatment and follow up often have to substitute for investigations as a gold standard, and are valuable tools for retrospective diagnosis. Bacteriological proof of either tuberculous or nontuberculous meningitis is available in only a small proportion of patients, because most patients have received antibiotics before hospitalisation, and the yield of CSF culture for mycobacteria/AFB stain is generally low. We find that a CT scan helps in differentiating tuberculous meningitis from other meningoencephalitides. Even in patients in whom polytherapy was started initially, diagnosis was usually clear after a CT scan. Some patients with pyogenic meningitis might respond initially to antituberculous treatment but would not show basal enhancement on CT. Basal enhancement has not been documented in numerous studies on pyogenic meningitis in the West, ${ }^{7-10}$ and this fact has also been pointed out by other authors. ${ }^{112}$ The other conditions that could produce such a CT picture are fungal meningitis, torulosis, neurosarcoidosis, and carcinomatous infiltration. ${ }^{112}$ These conditions would not improve on antituberculous treatment and thus would not be misallocated to the tuberculous meningitis group. Viral encephalitis or meningitis would also not be misdiagnosed as tuberculous meningitis because they are not known to produce basal enhancement. This picture of basal enhancement with or without tuberculoma is seen in about $90 \%$ of children with tuberculous meningitis. ${ }^{13} 14$

Twenty eight patients did not have a firm diagnosis, either because they died or their CT scan could not be done or did not show the characteristic features of basal enhancement/ tuberculoma. Such patients had to be treated as missing data and excluded from analysis because they did not satisfy the criteria for the diagnosis of either tuberculous or nontuberculous meningitis. This number constitutes $12 \%$ of the total patients and this 
proportion of incomplete data is inevitable in a study of this kind.

We found that $36 \%$ of patients with tuberculous meningitis had a predominant $(>50 \%)$ polymorphonuclear type of CSF pleocytosis at initial diagnosis. This is much higher than reported previously and this type of presentation is responsible for much of the initial diagnostic confusion. We find that it is not unusual to see patients with tuberculous meningitis with a polymorphonuclear predominant CSF pleocytosis, low CSF sugar, and even polymorphonuclear leucocytosis in the peripheral blood film. Patients with pyogenic meningitis, even if treated with oral or intramuscular antibiotics, usually have a polymorphonuclear CSF response. In our study, of the 94 patients with pyogenic meningitis, only five had a predominant lymphocytic response in CSF, and four of these had received intravenous antibiotics just before presentation.

On univariate analysis, 18 variables were significantly associated with the diagnosis of tuberculous meningitis. However, on multivariate analysis only five clinical/laboratory features showed significant independent association with the diagnosis (table 2). Presence of one or more of these features was seen in almost all patients with tuberculous meningitis. Thus, if none of these signs is present, the diagnosis of tuberculous meningitis becomes highly unlikely. When three or more of these signs were present, the sensitivity was $54.5 \%$ and specificity was $98.4 \%$, meaning that tuberculous meningitis was "ruled in" with a high degree of certainty. These values and the intermediate sensitivity and specificity levels achieved by the presence of two of these five scores (table 3) could be substantially improved by using the scoring system and ROC plot shown in fig 3.

Focal deficits were significantly more common in patients with tuberculous meningitis than other meningoencephalitides. Tuberculous meningitis produces an endarteritis and more frequent infarcts than other forms of central nervous system inflammation. $.^{15} \mathrm{Ex}-$ trapyramidal rigidity and movement disorders, decerebrate posturing, and optic neuritis were also seen more in tuberculous meningitis cases, perhaps because tuberculous meningitis pro- duces more basal exudates, therefore affecting basal structures such as basal ganglia, brain stem, and cranial nerves. ${ }^{3} 16$

Our study suggests that similar levels of accuracy can be obtained using clinical features as can be achieved with the use of complex laboratory investigations. This approach is certainly more cost effective where resources are limited. However, when differentiating dangerous but treatable disorders like tuberculous meningitis and pyogenic meningitis, a test with very high accuracy is needed, or the physician may be tempted to use both forms of treatment (conventional antibiotics and antituberculous drugs). Despite this consideration, this rule will at least guide physicians towards the diagnosis of tuberculous meningitis when one or more of these predictors are present and away from such a diagnosis when all are absent.

1 Upadhyaya GC, Tripathi BN, Shukla RK, Singh KN. Tuberculous meningitis in children. Indian $\mathcal{f}$ Pediatr 1984;51:633-6.

2 Daniel TM. New approaches to the rapid diagnosis of tuberculous meningitis. F Infect Dis 1987;155:599-603.

3 Udani PM. Neurotuberculosis in tuberculosis in children. Seth V, Puri RK, Sachdev HPS, eds. Delhi: Indian Academy of Pediatrics, 1991

4 Newton RW. Tuberculous meningitis. Arch Dis Child 1994; 70:364-6.

5 Corey L, Rubin RJ, Bregman D, et al. Diagnostic criteria for influenza $\beta$-associated Reye's syndrome: clinical vs pathologic criteria. Pediatrics 1977;60:702-8.

6 Mehta MN, Kumta NB, Bhanu KC, Deshpande DH. Tuberculous and pyogenic meningitis: a diagnostic diemma. Indian Pediatr 1975;12:1153-60.

7 Chaveria LE, du Boulay GH, Moseley IF. Intracranial infections: investigation by computerised axial tomography. Neuroradiology 1976;12:59-71.

8 Danziger A, Price H, Schechter M. An analysis of 113 intracranial infections. Neuroradiology 1980;19:31-42.

9 Cockrill HH, Dreisbach J, Lowe B, Yamauchi T. Computed tomography in intracranial infections. Am $\dot{f}$ Roentgenol

10 Bodino J, Pedro L, Del Valle M, et al. Computed tomography in purulent meningitis. Am 7 Dis Child 1982;136:495phy in

11 Bullock MMR, Welchman JM. Diagnostic and prognostic features of tuberculous meningitis on CT scanning. $\mathcal{F} \mathrm{Neu}-$ rol Neurosurg Psychiatry 1982;45:1098-101.

12 Nai-Shin C. Tuberculous meningitis: computed tomographic manifestations. Arch Neurol 1980;37:458-60.

13 Kumar R, Kohli N, Thavnani H, Kumar A, Sharma B. Value of CT scan in the diagnosis of meningitis. Indian Pediatr 1996;33:465-8.

14 Bhargava S, Gupta AK, Tandon PN. Tuberculous meningitis—a CT study. Br F Radiol 1982;55:189-96.

15 Molavi A, LeFrock JL. Tuberculous meningitis. Med Clin North Am 1985;69:315-31.

16 Kingsley DPE, Hendrickse WA, Kendall BE, Swash M, Singh V. Tuberculous meningitis: role of CT in management and prognosis. 7 Neurol Neurosurg Psychiatry 1987;50 $30-6$. 